\title{
Fotobiomodulação com laser de baixa potência na área de motricidade orofacial: uma análise comparativa a partir do conhecimento dos especialistas
}

\author{
Photobiomodulation with low-level laser therapy in the area of \\ orofacial motricity: comparative analysis from the knowledge of
} specialists

\author{
Maria Louize Justino Freire ${ }^{1}$ (D), Julyane Feitoza Coêlho ${ }^{2}$ (D), Paula Rayana Batista Correia ${ }^{1}$ (1), \\ Larissa Nadjara Alves Almeida ${ }^{3}$ (1), Leandro de Araújo Pernambuco' ${ }^{10}$, Giorvan Ânderson dos Santos Alves ${ }^{1}$
}

\begin{abstract}
RESUMO
Objetivo: comparar os conhecimentos e interesses dos fonoaudiólogos especialistas em motricidade orofacial quanto ao uso do laser de baixa potência antes e após a aprovação da Resolução ${ }^{\circ} 541$ do Conselho Federal de Fonoaudiologia. Métodos: trata-se de um estudo observacional, transversal e quantitativo, de caráter comparativo. Foi composto por dois momentos distintos: uma primeira coleta de dados, realizada em 2016, antes da aprovação da resolução e a segunda coleta, realizada em 2020, após a publicação das normativas. A primeira amostra foi constituída por 25 participantes e a segunda por 49 , todos especialistas em motricidade orofacial. O instrumento de coleta consistiu em um formulário online elaborado no Google Forms, composto por questões do perfil do fonoaudiólogo, conhecimentos, interesses e aplicabilidades da fotobiomodulação com o laser de baixa potência. Os dados foram analisados utilizando estatística descritiva e inferencial. Resultados: nos dois momentos, a expressiva maioria dos profissionais indicou saber o que é fotobiomodulação e concordou que o especialista em motricidade orofacial pode atuar com a técnica. Além disso, os profissionais apontaram a disfunção temporomandibular e a paralisia facial como principais casos para aplicações. Foram encontradas diferenças estatisticamente significativas entre os resultados obtidos antes e após a publicação da resolução, verificando-se aumento expressivo no acesso à bibliografia, capacitação, conhecimento das normas de biossegurança, acesso ao aparelho de laser e utilização na prática clínica. Conclusão: constatou-se mudança no perfil profissional em relação ao uso da fotobiomodulação, com aumento dos conhecimentos, interesses e aplicabilidades pelos especialistas em motricidade orofacial, após a publicação da resolução normativa.
\end{abstract}

Keywords: Fonoaudiologia; Fonoterapia; Lasers; Terapia a laser; Terapia com luz de baixa intensidade

\begin{abstract}
Purpose: To compare knowledge and interest of speech therapists specialized in Orofacial Motricity regarding the use of low-level laser before and after approval of resolution No. 541 by the Brazilian Federal Speech Language Pathology and Audiology Council (CFFa). Methods: Cross-sectional observational and quantitative study of comparative character consisting of two distinct moments: first data collection performed in 2016, before approval of the resolution; and second, collection performed in 2020, after publication of the regulation. The first sample consisted of 25 participants and the second of 49 , with the entire sample consisting of specialists in Orofacial Motricity. The collection instrument was an online Google Form consisting of questions on the profile of the speech therapist, knowledge, interests, and applicability of photobiomodulation with low-level laser. Data were analyzed using descriptive and inferential statistics. Results: In both moments, the significant majority of professionals indicated knowing what is photobiomodulation, agreed that the Orofacial Motricity specialist can act with the technique and indicated temporomandibular dysfunction and facial paralysis as main applications. Statistically significant differences were found between the results obtained before and after the publication of the resolution, with a significant increase in access to bibliography, training, knowledge of biosafety standards, access to the low-level laser apparatus and use in clinical practice. Conclusion: Change in the professional profile regarding the use of photobiomodulation was verified, with increased knowledge, interest, and applicability by Orofacial Motricity specialists after publication of the normative resolution.
\end{abstract}

Keywords: Speech, language and hearing sciences; Speech therapy; Lasers; Laser therapy; Low-level light therapy

\footnotetext{
Trabalho realizado na Universidade Federal da Paraíba - UFPB - João Pessoa (PB), Brasil.

${ }^{1}$ Departamento de Fonoaudiologia, Universidade Federal da Paraíba - UFPB - João Pessoa (PB), Brasil.

${ }^{2}$ Programa de Pós-graduação em Linguística, Universidade Federal da Paraíba - UFPB - João Pessoa (PB), Brasil

${ }^{3}$ Centro Universitário de João Pessoa - UNIPÊ - João Pessoa (PB), Brasil.

Conflito de interesses: Não. da orientação da pesquisa.

Financiamento: Nada a declarar.

Autor correspondente: Giorvan Ânderson dos Santos Alves. E-mail: anderson_ufpb@yahoo.com.br

Recebido: Maio 12, 2021; Aceito: Junho 13, 2021
}

Contribuição dos autores: MLJF contribuiu na aquisição, análise e interpretação dos dados, além da redação do artigo; JFC contribuiu com a revisão crítica final da versão a ser apresentada para publicação; PRBC contribuiu na aquisição, análise e interpretação dos dados; LNAA contribuiu no delineamento do estudo e análise dos dados; LAP contribuiu com a revisão crítica de informações intelectuais relevantes; GÂSA contribuiu na concepção e delineamento do estudo, além 


\section{INTRODUÇÃO}

As propriedades terapêuticas do laser de baixa potência (LBP) têm sido bastante estudadas, por ser um recurso de fácil manuseio, não invasivo, que possui ação analgésica, antiinflamatória e regenerativa ${ }^{(1,2)}$. No campo da Fonoaudiologia, os estudos revelam que os seus efeitos têm se mostrado bastante positivos, quando associados aos tratamentos convencionais, podendo ser um importante aliado na clínica de motricidade orofacial $(\mathrm{MO})^{(3)}$.

A MO corresponde a uma das áreas da ciência fonoaudiológica, responsável pelo estudo, avaliação, pesquisa, reabilitação e aperfeiçoamento das funções estomatognáticas ${ }^{(4)}$. A prática clínica na área está cada vez mais baseada em evidências científicas, o que proporciona maior êxito nas intervenções. Nessa perspectiva, observa-se um aumento de trabalhos científicos demonstrando os benefícios do LBP e seus resultados dentro das terapias fonoaudiológicas, principalmente estudos com a disfunção temporomandibular (DTM) $)^{(3,5,6)}$.

Em razão dos diversos benefícios apresentados, o LBP tem se mostrado um importante recurso complementar na terapia, facilitando o processo de reabilitação do paciente e contribuindo para a adequação de estruturas e funções estomatognáticas. De acordo com as propriedades apresentadas pelo LBP, sua ação se dá por meio de modificações diretas nas células, resultado de efeitos fotofísicos, fotoquímicos e fotobiológicos ${ }^{(7)}$. Esses benefícios são observados na área da $\mathrm{MO}$, sobretudo quando somados o LBP e a terapia convencional, proporcionando um diferencial no tratamento de pacientes com desordem temporomandibular, paralisia facial e patologias que necessitem de cicatrização tecidual ${ }^{(6,8,9)}$.

O Conselho Federal de Fonoaudiologia (CFFa) aprovou, em março de 2019, por meio da Resolução n ${ }^{\circ} 541^{(10)}$, o LBP como um recurso a ser utilizado pelos fonoaudiólogos em sua prática clínica. As normativas foram elaboradas a partir de consulta e considerando o parecer da Associação Brasileira de Motricidade Orofacial (ABRAMO) e do Departamento de Motricidade Orofacial da Sociedade Brasileira de Fonoaudiologia (SBFa). Diante das disposições do documento, o profissional se torna habilitado a fazer uso dessa tecnologia após capacitação/ especialização.

Antes da aprovação dessa resolução, um levantamento realizado com especialistas em MO apontou que a maioria dos participantes não utilizava o LBP em sua prática clínica, não obteve conhecimentos sobre o assunto na graduação, nem na pós-graduação, mas concordava que os fonoaudiólogos deveriam adotar essa nova técnica em seus procedimentos, como um recurso complementar ao tratamento ${ }^{(11)}$. Contudo, não se dispõe de estudos sobre o conhecimento dos fonoaudiólogos especialistas em MO após a publicação da resolução do CFFa, em 2019.

Nesse sentido, considerando que as recomendações poderiam influenciar a prática profissional em relação ao uso do recurso e buscando identificar as possíveis mudanças advindas com a publicação das normativas oficiais da profissão, o objetivo do presente estudo foi comparar os conhecimentos e interesses dos fonoaudiólogos brasileiros especialistas em MO quanto ao uso do LBP, antes e após a aprovação da Resolução n ${ }^{0} 541$ do CFFa.

\section{MÉTODO}

O estudo é do tipo observacional, longitudinal e quantitativo, de caráter comparativo. Foi composto por dois momentos distintos: uma primeira coleta de dados, realizada em 2016, antes da aprovação da resolução do CFFa e a segunda coleta, realizada em 2020, após a publicação das normativas.

A primeira amostra foi composta por 25 participantes e a segunda por 49, todos especialistas em motricidade orofacial, de todo o Brasil, elegidos por conveniência, tendo como critério de inclusão ser profissional graduado na área de Fonoaudiologia e especialista em $\mathrm{MO}^{(11)}$.

A pesquisa foi conduzida por meio da divulgação de um questionário online via redes sociais (Facebook e Instagram) e aplicativo de mensagens (Whatsapp). Durante o período de quatro semanas, todas as sextas-feiras foi compartilhado um folder da pesquisa juntamente com o link que direcionou o voluntário para o site Google Forms, que continha o Termo de Consentimento Livre Esclarecido (TCLE) e o questionário do estudo.

Todos os critérios éticos foram considerados, o estudo foi previamente submetido à avaliação e aprovação (parecer $\mathrm{n}^{\circ}$ 4.103.029) do Comitê de Ética em Pesquisa com Seres Humanos da Universidade Federal da Paraíba - UFPB, para posterior início das atividades de coleta. Todos os voluntários do estudo assinaram o TCLE, concordando em participar.

As respostas dos voluntários ao questionário, que continha perguntas sobre o perfil do fonoaudiólogo, dados de formação, atuação profissional e conhecimentos sobre a fotobiomodulação, constituíram os dados do primeiro estudo realizado em 2016 e da presente pesquisa. Esse questionário foi adaptado e composto por 34 questões, sendo 20 replicadas do estudo original, com a permissão dos autores, e inseridas mais 14 questões específicas. Entretanto, nesta analise foram consideradas apenas as perguntas coincidentes nos dois estudos.

Os pesquisadores tiveram acesso ao banco de dados da pesquisa anteriormente realizada ${ }^{(11)}$, permitindo um estudo comparativo. Para análise dos dados, foi realizada análise estatística descritiva, a partir de medidas de frequência absoluta e relativa, assim como estatística inferencial, utilizando o teste Qui-quadrado de Pearson, considerando a significância de $1 \%$.

\section{RESULTADOS}

Em relação ao perfil profissional, alguns aspectos divergiram nos dois momentos de coleta. No primeiro momento, a maioria dos participantes era de doutores e docentes; já no segundo momento, os participantes eram, principalmente, especialistas. Contudo, nas duas amostras, verificou-se a mesma média de idade, o tempo de profissão predominante foi de 21 anos ou mais e o principal local de atuação foi a clínica. Os dados de caracterização da amostra estão expostos no Quadro 1.

No que se refere aos conhecimentos sobre o recurso, nos dois momentos, a expressiva maioria dos participantes indicou saber o que é fotobiomodulação, embora não tivesse adquirido conteúdos sobre a temática introduzidos na graduação ou pósgraduação. 
Quadro 1. Caracterização dos participantes, seus conhecimentos e interesses sobre fotobiomodulação antes e após a resolução do Conselho Federal de Fonoaudiologia

\begin{tabular}{|c|c|c|}
\hline Variáveis & Antes da resolução do CFFa & Após a resolução do CFFa \\
\hline \multirow[t]{2}{*}{ Faixa etária (média) } & 43 & 43,2 \\
\hline & Doutorado $(61,5 \%)$ & Doutorado $(28,6 \%)$ \\
\hline \multirow[t]{4}{*}{ Principal titulação } & Mestrado $(26,9 \%)$ & Mestrado $(16,3 \%)$ \\
\hline & Especialização (11,5\%) & Especialização $(36,7 \%)$ \\
\hline & Até 1 ano $(0 \%)$ & Até 1 ano $(2,0 \%)$ \\
\hline & De 1 a $5(0 \%)$ & De 1 a $5(10,2 \%)$ \\
\hline \multirow[t]{4}{*}{ Tempo de profissão } & De 5 a $10(3,8 \%)$ & De 5 a $10(4,1 \%)$ \\
\hline & De 10 a $20(42,3 \%)$ & De 10 a $20(36,7 \%)$ \\
\hline & 21 anos ou mais $(53,8 \%)$ & 21 anos ou mais $(46,9 \%)$ \\
\hline & $\begin{array}{l}\text { Clínica e/ou estágio }(19,2 \%) \text {; sala de aula } \\
(11,5 \%) \text {; outros locais }(7,7 \%)\end{array}$ & $\begin{array}{l}\text { Clínica }(75,5 \%) \text {; home care }(32,7 \%) \text {; hospitais } \\
(22,4 \%) \text {; sala de aula }(20,4 \%)\end{array}$ \\
\hline Locais de atuação & Docentes $(81,5 \%)$ & Docentes $(49,0 \%)$ \\
\hline \multirow[t]{3}{*}{ Docência } & Leciona na graduação $(23,1 \%)$ & Leciona na graduação $(8,2 \%)$ \\
\hline & Leciona na pós-graduação $(30,8 \%)$ & Leciona na Pós-graduação $(22,4 \%)$ \\
\hline & Leciona em ambos $(34,6 \%)$ & Leciona em ambos $(18,4 \%)$ \\
\hline Sabe o que é fotobiomodulação & $\operatorname{Sim}(92,3 \%)$ & $\operatorname{Sim}(100 \%)$ \\
\hline $\begin{array}{l}\text { Conteúdo sobre fotobiomodulação introduzido } \\
\text { na graduação }\end{array}$ & $100 \%$ dos profissionais não tiveram & $93,9 \%$ dos profissionais não tiveram \\
\hline $\begin{array}{l}\text { Conteúdo sobre fotobiomodulação introduzido } \\
\text { na pós-graduação }\end{array}$ & $96,2 \%$ não tiveram & $85,7 \%$ não tiveram \\
\hline \multirow[t]{3}{*}{ Modo de acesso a um aparelho de LBP } & Aparelho particular (0\%) & Aparelho particular $(63,3 \%)$ \\
\hline & Universidades $(23,1 \%)$ & Universidades $(12,2 \%)$ \\
\hline & & Aluguel/empréstimo $(6,1 \%)$. \\
\hline \multirow{4}{*}{$\begin{array}{l}\text { Concordam que o especialista em MO deve } \\
\text { atuar em fotobiomodulação }\end{array}$} & $\operatorname{Sim}(76,9 \%)$ & $\operatorname{Sim}(67,3 \%)$ \\
\hline & Não $(3,8 \%)$ & Não $(2,04 \%)$ \\
\hline & Não sabe opinar $(19,2 \%)$ & Não sabe opinar $(30,76 \%)$ \\
\hline & DTM $(46,2 \%)$ & DTM $(28,57 \%)$ \\
\hline \multirow[t]{2}{*}{ Indicações terapêuticas } & Paralisia Facial $(3,8 \%)$ & Paralisia Facial (40\%) \\
\hline & Ambas $(34,6 \%)$ & \\
\hline
\end{tabular}

Legenda: $\mathrm{CFFa}$ = Conselho Federal de Fonoaudiologia; $\mathrm{LBP}$ = Laser de baixa potência; $\mathrm{MO}$ = motricidade orofacial; $\mathrm{DTM}=$ disfunção temporomandibular

Tabela 1. Associações entre os conhecimentos e interesses dos especialistas antes e após a resolução do Conselho Federal de Fonoaudiologia

\begin{tabular}{|c|c|c|c|c|c|c|c|}
\hline & \multicolumn{3}{|c|}{ Antes da resolução do CFFa } & \multicolumn{3}{|c|}{ Após a resolução do CFFa } & \multirow{2}{*}{ Valor de $p$} \\
\hline & Resposta & $\mathbf{N}$ & $\%$ & Resposta & $\mathbf{N}$ & $\%$ & \\
\hline \multirow{2}{*}{$\begin{array}{l}\text { Fez alguma formação/capacitação em } \\
\text { fotobiomodulação }\end{array}$} & Sim & 2 & $7,7 \%$ & Sim & 41 & $83,7 \%$ & \multirow{2}{*}{$\mathrm{p}=0,0001^{*}$} \\
\hline & Não & 24 & $92,3 \%$ & Não & 8 & $16,3 \%$ & \\
\hline $\begin{array}{l}\text { Acesso a alguma bibliografia em } \\
\text { fotobiomodulação }\end{array}$ & Não & 16 & $61,5 \%$ & Não & 8 & $16,3 \%$ & $p=0,0001^{*}$ \\
\hline Acesso a um aparelho de LBP & Sim & 5 & $19,2 \%$ & $\operatorname{Sim}$ & 41 & $83,7 \%$ & $\mathrm{p}=0,0001^{*}$ \\
\hline Utiliza o LBP na prática clínica & Não & 25 & $96,2 \%$ & Não & 14 & $28,6 \%$ & $p=0,0001^{*}$ \\
\hline \multirow{2}{*}{$\begin{array}{l}\text { Conhece as normas de } \\
\text { biossegurança em fotobiomodulação }\end{array}$} & Sim & 6 & $23,1 \%$ & Sim & 42 & $85,7 \%$ & \multirow{2}{*}{$p=0,0001^{*}$} \\
\hline & Não & 20 & $76,9 \%$ & Não & 7 & $14,3 \%$ & \\
\hline
\end{tabular}

Teste Qui-quadrado de Pearson, $p<0,01^{*}$

Legenda: CFFa = Conselho Federal de Fonoaudiologia; LBP = Laser de baixa potência; N = Número de participantes; \%= percentual

Observou-se um número expressivamente maior de participantes no segundo momento da coleta, o que pode estar relacionado à forma de divulgação, pois, inicialmente, foram utilizados apenas convites por e-mail e, posteriormente, optouse pelas redes sociais. Os conhecimentos e interesses sobre fotobiomodulação, antes e após a resolução do CFFa, também estão apresentados no Quadro 1.
Foram verificadas diferenças estatisticamente significativas $(p<0,01)$ entre os resultados obtidos antes e após a resolução do $\mathrm{CFFa}$, sendo possível observar que, atualmente, mais pessoas têm procurado se capacitar em fotobiomodulação, têm acesso à bibliografia sobre LBP, conhecem as normas de biossegurança, têm acesso a um equipamento de LBP e utilizam o recurso na prática clínica (Tabela 1). 


\section{DISCUSSÃO}

Tendo em vista que o estudo foi realizado em duas etapas distintas, antes e após o estabelecimento das normativas para a utilização do LBP por fonoaudiólogos, a análise dos resultados permitiu o melhor entendimento das modificações advindas com a publicação da resolução do CFFa. Foi observada uma mudança no perfil profissional em relação ao uso da técnica de fotobiomodulação.

A diferença verificada nas características dos participantes, nas duas amostras de coleta de dados, pode ser resultante da forma de divulgação do questionário, que, inicialmente, foi encaminhado apenas por $e$-mail para os especialistas em MO, recrutados por meio de dados disponibilizados pela ABRAMO. Já no segundo momento, a divulgação foi mais ampla, utilizando também as redes sociais como estratégia para alcançar o maior número de profissionais, de todas as regiões do país. Um aspecto interessante é que mesmo no primeiro momento os profissionais sendo predominantemente doutores e docentes; e no segundo, principalmente especialistas, observou-se o aumento nas informações, interesses e acesso ao recurso após a publicação da resolução do CFFa.

A realização de formação/capacitação sobre fotobiomodulação em Fonoaudiologia ou saúde, assim como o acesso a alguma bibliografia referente à temática, foram alguns dos resultados que apresentaram aumento significativo entre os dois momentos, revelando que houve maior procura dos profissionais a respeito da técnica.

Tais achados podem estar relacionados com a ampliação do número de publicações na área, resultado dos novos estudos que têm abordado a fotobiomodulação ${ }^{(6,12)}$, como também podem estar associados a maior disponibilidade de cursos de formação em fotobiomodulação. Ainda, podem estar relacionados à recente aprovação da técnica pelo CFFa, fato que levou ao aumento do conhecimento sobre o recurso, verificado por meio da comparação entre os dois momentos de coleta deste estudo. Após a resolução do CFFa, houve crescimento do interesse sobre a temática, o que pode estar relacionado à obrigatoriedade da qualificação dos profissionais previamente à utilização do recurso.

Embora o equipamento tenha um custo relativamente alto, o acesso ao aparelho se tornou maior, quando comparados os dois momentos do estudo. Inicialmente, os participantes dispunham do recurso apenas nas universidades e, atualmente, mais da metade dos pesquisados dispõe de forma particular. Pôde-se verificar, também, mediante análise comparativa, que a utilização desse recurso na prática clínica em MO foi significativamente ampliada. Nesse sentido, observa-se que a publicação das normativas oficiais da profissão contribuiu para o maior conhecimento, valorização e incremento na utilização do LBP por fonoaudiólogos.

Atualmente, a maioria dos pesquisados consente que os especialistas em MO podem atuar com a técnica de fotobiomodulação, aspecto que pode estar vinculado à disseminação dos estudos e evidências clínicas na área, visto que a fotobiomodulação apresenta resultados satisfatórios em pacientes que necessitam de cuidados envolvendo o campo da MO. Nessa perspectiva, vale destacar que essa é uma das áreas da Fonoaudiologia que mais apresenta respaldos científicos na utilização da técnica ${ }^{(6,9,13)}$.

No presente estudo, as principais aplicações clínicas apontadas foram em casos de paralisia facial e DTM, o que está de acordo com discussões e evidências científicas na área de $\mathrm{MO}^{(5,9,14)}$. No que se refere à paralisia facial, estudos trazem resultados positivos quanto à associação do recurso ao tratamento convencional, mostrando melhoras significativas em pacientes com paralisia facial idiopática. Nesses casos, a utilização do LBP trouxe vantagens relacionadas à redução do período de tratamento, gerando mais agilidade e eficácia ${ }^{(9,12)}$.

O uso do LBP na DTM vem sendo consolidado por meio de práticas clínicas e de pesquisas que mostram a sua ação benéfica, tornando as evidências científicas mais fortes. Achados demonstram que o LBP atua na redução de quadros álgicos provocados pela DTM, devido a sua ação analgésica e anti-inflamatória, aumentando, também, a amplitude dos movimentos mandibulares, quando associado a outros métodos terapêuticos convencionais ${ }^{(5,6,8,15)}$.

Por fim, outro aspecto a ser destacado é o acesso às normas de biossegurança para utilização do recurso. No primeiro momento do estudo, verificou-se que grande parte dos pesquisados não tinha acesso a essas normas para utilização do LBP. Já no momento atual, foi observada uma tendência oposta, ou seja, a maioria dos especialistas demonstra conhecimento dessas normas, tornando esse um achado positivo e de grande relevância, tendo em vista que é um quesito importante para a aplicação do LBP e que demanda atenção especial do terapeuta para o uso adequado dos equipamentos de proteção individual ${ }^{(1)}$.

Diante do exposto, observa-se mudança no perfil dos profissionais especialistas em MO após a publicação da Resolução $\mathrm{n}^{\mathrm{o}} 541$ do CFFa, com aumento dos conhecimentos, interesses e utilização clínica, envolvendo as diversas aplicabilidades do LBP. Considerando o contexto atual da prática fonoaudiológica baseada em evidências, observam-se avanços com a ampliação das pesquisas científicas em fotobiomodulação na área de MO, proporcionando maior confiabilidade na utilização da técnica. Em conformidade com as normativas da profissão, destaca-se a necessidade da formação/capacitação mínima dos fonoaudiólogos para atuarem com esse recurso, considerando as especificidades e peculiaridades envolvidas no domínio da técnica e o compromisso ético dos profissionais na busca de ofertar as melhores intervenções, alcançando, assim, resultados clínicos satisfatórios.

\section{CONCLUSÃO}

A partir da publicação da resolução do CFFa, houve um crescimento expressivo de profissionais que tiveram interesse na fotobiomodulação, buscando conhecer e se aprimorar para utilizar a técnica. Juntamente com o avanço das pesquisas, que revelam as potencialidades terapêuticas do LBP, a regulamentação promoveu o aumento do interesse e da confiança dos fonoaudiólogos, repercutindo em maior procura, utilização clínica e ampliação do acesso aos aparelhos de laser.

\section{REFERÊNCIAS}

1. Lizarelli RFZ. Protocolos clínicos odontológicos: uso do laser de baixa intensidade. 4. ed. São Paulo: MM Optics Ltda; 2010.

2. de Freitas LF, Hamblin MR. Proposed mechanisms of photobiomodulation or low-level light therapy. IEEE J Sel Top Quantum Electron. 2016 MaioJun;22(3):7000417. http://dx.doi.org/10.1109/JSTQE.2016.2561201. PMid:28070154. 
3. Machado BC, Mazzetto MO, Da Silva MA, de Felício CM. Effects of oral motor exercises and laser therapy on chronic temporomandibular disorders: a randomized study with follow-up. Lasers Med Sci. 2016;31(5):945-54. http://dx.doi.org/10.1007/s10103-016-1935-6. PMid:27085322.

4. Motta AR, Medeiros AMC, Berretin-Felix G, Folha GA, Genaro KF, Lucena MM, et al. Áreas de domínio do especialista em Motricidade Orofacial. In: Rahal A, Motta AR, Fernandes CG, Cunha DA, Migliorucci RR, Berretin-Felix G, organizadores. Manual de motricidade orofacial. São José dos Campos-SP: Pulso editorial; 2014.

5. Melchior MO, Brochini APZ, Silva MAMR. Low-level laser therapy associated to occlusal splint to treat temporomandibular disorder: controlled clinical trial. Rev Dor. 2017 Mar;18(1):12-7. http://dx.doi org/10.5935/1806-0013.20170004.

6. Batista SL, Coêlho JF, Almeida LNA, Spinelli-Pessoa L, Vasconcelos ML, Alves GAS. Oral amplitude and orofacial pain in patients with temporomandibular dysfunction submitted to lasertherapia and a miofacional orofacial therapy. Rev Bras Ciênc Saúde. 2019 Dec;23(2):8594. http://dx.doi.org/10.22478/ufpb.2317-6032.2019v23n2.48415.

7. Hamblin MR. Mechanisms and mitochondrial redox signaling in photobiomodulation. Photochem Photobiol. 2018;94(2):199-212. http://dx.doi.org/10.1111/php.12864. PMid:29164625.

8. Gomes CF, Schapochnik A. O uso terapêutico do LASER de Baixa Intensidade (LBI) em algumas patologias e sua relação com a atuação na Fonoaudiologia. Distúrb Comun. 2017 Set 29;29(3):570-8. http:// dx.doi.org/10.23925/2176-2724.2017v29i3p570-578.

9. Vanderlei T, Bandeira RN, Canuto MSB, Alves GAS. Laserterapia de baixa potência e paralisia facial periférica: revisão integrativa da literatura.
Terapia a laser e Paralisia de Bell. Distúrb Comun. 2019 Dez;31(4):55764. http://dx.doi.org/10.23925/2176-2724.2019v31i4p557-564.

10. Brasil. Conselho Federal de Fonoaudiologia. Resolução CFFa n ${ }^{0} 541$, de 15 de março de 2019 Dispõe sobre o uso do recurso de Laser de Baixa Intensidade- LBI por fonoaudiólogos. Diário Oficial da União [Internet]; Brasília; 2019 [citado em 2020 Set 1]. Disponível em: https://www.fonoaudiologia.org.br/resolucoes/

11. Matos AS, Berretin-Felix G, Bandeira RN, Lima JAS, Almeida LNA, Alves GAS. Laser therapy applied to orofacial motricity: perception of members of the Brazilian Orofacial Motricity Association-Abramo. Rev CEFAC. 2018 Fev;20(1):61-8. http://dx.doi.org/10.1590/1982-021620182017317.

12. Ordahan B, Karahan AY. Role of low-level laser therapy added to facial expression exercises in patients with idiopathic facial (Bell's) palsy. Lasers Med Sci. 2017 Maio;32(4):931-6. http://dx.doi.org/10.1007/ s10103-017-2195-9. PMid:28337563.

13. Alves VMN, Furlan RMMM, Motta AR. Immediate effects of photobiomodulation with low-level laser therapy on muscle performance: an integrative literature review. Rev CEFAC. 2019;21(4):e12019. http://dx.doi.org/10.1590/1982-0216/201921412019.

14. Poloni MM, Marques NP, Ribeiro NV Jr, Sperandio FF, Hanemann JAC, de Carli ML. Bell's palsy treated with photobiomodulation in an adolescent: rare case report and review of the published literature. Int J Paediatr Dent. 2018;28(6):658-62. http://dx.doi.org/10.1111/ipd.12424. PMid:30218464.

15. Costa SAP, Florezi GP, Artes GE, Costa JR, Gallo RT, Freitas PM, et al. The analgesic effect of photobiomodulation therapy $(830 \mathrm{~nm})$ on the masticatory muscles: a randomized, double-blind study. Braz Oral Res. 2017;31(0):e107. http://dx.doi.org/10.1590/1807-3107bor-2017. vol31.0107. PMid:29267668. 